\title{
Urinary Metabolites as Biomarkers for Diagnosis of Breast Cancer: A Preliminary Study
}

\author{
Jiwon Park, M.D. ${ }^{1}$, Yumi Shin, M.S. ${ }^{2}$, Tae Hyun Kim, M.D., Ph.D. ${ }^{1}$, Dong-Hyun Kim, Ph.D. ${ }^{2}$, \\ Anbok Lee, M.D., Ph.D. ${ }^{1}$ \\ ${ }^{1}$ Department of Surgery, Busan Paik Hospital, College of Medicine, Inje University, Busan; ${ }^{2}$ Department of Pharmacology, College of Medicine, Inje \\ University, Busan, Korea
}

Purpose: For early detection of breast cancer, tests with high sensitivity and specificity are needed. Metabolomics, the study of chemical processes involving metabolites, can be used to identify diagnostic biomarkers for a variety of types of cancers. In this study we identified biomarkers of breast cancer by profiling urinary metabolites. Methods: We performed metabolite profiling of 30 urine samples from 14 patients with breast cancer and 16 healthy controls by liquid chromatography-mass spectrometry. An orthogonal partial least squares-discriminant analysis (OPLS-DA), Student's t-test, and receiver operating characteristic (ROC) analysis were performed to identify metabolites that were potential diagnostic biomarkers for breast cancer. Results: The OPLS-DA showed clear separation between the two groups. Of the 95 metabolites detected, 24 potential biomarkers were identified by Student's t-test. A ROC analysis showed that concentrations of N-(2-furoyl) glycine, histidine, and D-tagarose were significantly higher (area under the ROC curve $[A U C]>0.7$ ) and those of trigonellinamide, L-galacto-2-heptulose, creatinine, and xanthine were significantly lower (AUC $\geq 0.8$ ) in the patients with breast cancer than in the healthy controls. Conclusion: Measurement of the concentrations of urinary metabolites can be used to screen for early breast cancer. We plan to explore diagnostic biomarkers of breast cancer in blood and urine further in a larger study.

Key Words: Breast neoplasms, Biomarkers, Mass spectrometry, Urine

\section{INTRODUCTION}

Early detection of breast cancer is important for successful treatment and a good prognosis. Early-stage breast cancer is typically asymptomatic - there is no lump or pain. Therefore, breast cancer screening is extremely important.

Mammography is the gold standard for breast cancer screening but has a low sensitivity of 54-77\%, depending on the type of mammography [1]. Mammography is also inconvenient and involves exposure to radiation. Therefore, noninvasive screening tests for breast cancer that have high sensitivity and specificity are needed.

Metabolomics is an emerging field that enables identification of the

Correspondence: Dong-Hyun Kim, Ph.D.

Department of Pharmacology, College of Medicine, Inje University, 75 Bokji-ro, Busanjin-gu, Busan 47392, Korea

Tel: +82-51-890-6411, Fax: +82-51-893-1232, E-mail: dhkim@inje.ac.kr

Correspondence: Anbok Lee, M.D., Ph.D.

Department of Surgery, Busan Paik Hospital, College of Medicine, Inje University, 75 Bokji-ro, Busanjin-gu, Busan 47392, Korea

Tel: +82-51-890-6859, Fax: +82-51-898-9427, E-mail: ab-lee@hanmail.net

Received:Sep 1, 2019 Revised:Sep 13, 2019 Accepted: Nov 3, 2019 key metabolic pathways in various types of cancers. The metabolism of proliferating cancer cells differs from that of normal differentiated cells [2]. In cancer tissue, hypoxic metabolism is typically upregulated, and lactate is produced; this is known as the Warburg effect [3-5]. The amino acid glutamine provides carbon to enable rapid proliferation of cancer cells and participates in nucleic-acid synthesis [6,7]. Metabolomics studies have typically focused on plasma, serum, urine, tissue, and cerebrospinal fluid specimens.

Urine has long been used for diagnostic purposes, based on its smell, color, and acidity [8]. It is the final product of metabolism and is closely related to clinical phenotype. However, it remains unclear whether the levels of metabolites in blood reflect direct leakage from tumors. If leakage into blood can be a primary reason for metabolic change, the same is true for urine, which reflects one further step in metabolic transformation [9]. Mass spectrometry (MS)-based metabolomics is a useful tool for profiling changes in metabolites in urine [10].

We plan to explore plasma and urinary biomarkers of breast cancer using liquid chromatography-mass spectrometry (LC-MS). Here, we 
performed LC-MS analysis of urine samples to identify endogenous metabolites that might distinguish patients with breast cancer from healthy controls.

\section{METHODS}

\section{Selection of patients and collection of urine samples}

This study included 14 patients with breast cancer and 16 healthy controls. The patients with breast cancer were diagnosed and surgically treated at Inje University Busan Paik hospital between 2011 and 2016. Patients who received neoadjuvant chemotherapy were excluded. The healthy controls were individuals with no history of other diseases, including malignancies. The study protocol was approved by the Institutional Review Board of Inje University Busan Paik hospital (IRB No. 17-0191).

\section{Chemicals and reagents}

Cholic acid-d5 (internal standard) was purchased from Toronto Research Chemicals (Toronto, Canada). Urease was purchased from Sigma-Aldrich (St. Louis, USA), and high-performance liquid chromatography (HPLC)-grade acetonitrile, methanol, and water were purchased from JT Baker (Philipsburg, USA). Other chemicals were of the highest grade commercially available.

\section{Sample preparation}

Urease $(15 \mathrm{U})$ was added to $50-\mu \mathrm{L}$ urine samples and incubated at $37^{\circ} \mathrm{C}$ for 15 min to eliminate excess urea. Next, methanol $(50 \mu \mathrm{L})$ containing $5 \mu \mathrm{g} / \mathrm{mL}$ cholic acid-d5 (internal standard) was added to the urine samples. The mixture was vortexed and centrifuged at 13,200 rpm for $5 \mathrm{~min}$ at $4^{\circ} \mathrm{C}$. The supernatant $(100 \mu \mathrm{L})$ was diluted with 100 $\mu \mathrm{L}$ of distilled water in a fresh tube and vortexed; finally, the supernatant was decanted and injected onto the HPLC column.

\section{Chromatographic separation and mass analysis}

The samples were analyzed using an Agilent 6530 True High Definition quadrupole time-of-flight mass spectrometer (Agilent, Santa Clara, USA) coupled to a 1200-series HPLC system (Agilent). Separation was performed on a BEH C18 column $(100 \times 2.1 \mathrm{~mm}, 1.7 \mu \mathrm{m}$; Waters, Milford, USA $)$ and a ZIC-HILIC column $(100 \times 2.1 \mathrm{~mm}, 3.5 \mu$ m; Merck, Darmstadt, Germany). For the BEH C18 column, the mo- bile phase was $0.1 \%$ formic acid in water (A) and $0.1 \%$ formic acid in acetonitrile (B) at a flow rate of $0.4 \mathrm{~mL} / \mathrm{min}$. The gradient was as follows: $2 \%$ mobile phase B for $1 \mathrm{~min}$, an increase to $20 \%$ at $3 \mathrm{~min}$ and an increase to $90 \%$ at $8 \mathrm{~min}$, which was maintained for $6 \mathrm{~min}$; the total run time was $18 \mathrm{~min}$. The temperatures of the column and autosampler were $35^{\circ} \mathrm{C}$ and $4^{\circ} \mathrm{C}$, respectively, and the injection volume was $3 \mu$ L. For the ZIC-HILIC column, the mobile phase was $10 \mathrm{mM}$ ammonium acetate in 5/95 acetonitrile/water (A) and $10 \mathrm{mM}$ ammonium acetate in 95/5 in acetonitrile/water (B), at a flow rate of $0.5 \mathrm{~mL} / \mathrm{min}$. The gradient was as follows: 99\% mobile phase B for 1 min followed by a decrease to $50 \%$ at $15 \mathrm{~min}$, which was maintained for $2 \mathrm{~min}$; the total run time was $22 \mathrm{~min}$. The temperatures of the column and autosampler were $40^{\circ} \mathrm{C}$ and $4^{\circ} \mathrm{C}$, respectively, and the injection volume was $5 \mu \mathrm{L}$. Electrospray ionization (ESI) was performed in both the positive- and negative ion modes for the BEH column and in the negative-ion mode for the ZIC-HILIC column. The sheath-gas flow rate was set at $11 \mathrm{~L} / \mathrm{min}$. The drying-gas flow rate was set at $12 \mathrm{~L} / \mathrm{min}$ at $350^{\circ} \mathrm{C}$. The nebulizer temperature was maintained at $350^{\circ} \mathrm{C}$. The capillary voltage was set at $4,000 \mathrm{~V}$ in positive ion mode and $-4,000 \mathrm{~V}$ in negative ion mode, and the fragmentor was set at $110 \mathrm{~V}$. The data were acquired in a scan range from $\mathrm{m} / \mathrm{z} 50$ to 1,000 in centroid mode. A reference compound $\left(\mathrm{C}_{18} \mathrm{H}_{18} \mathrm{O}_{6} \mathrm{~N}_{3} \mathrm{P}_{3} \mathrm{~F}_{24} ;[\mathrm{M}+\mathrm{H}]^{+}\right.$ $=922.0098$ and $\left.[\mathrm{M}+\text { formate }]^{-}=966.0007\right)$ was used to correct masses during analysis. An automatic MS/MS analysis was performed for peak identification, and the collision energy was set at $30 \mathrm{eV}$.

\section{Data processing}

MS data were obtained using the following parameters: retention time range, 0.5 to $18 \mathrm{~min}$ and 0.5 to $22 \mathrm{~min}$ for the BEH and ZIC-HILIC columns, respectively; mass range, $m / z 50$ to 1,000 ; and extracted ion chromatogram window, $0.02 \mathrm{Da}$. The data were converted to mzXML format using conversion software. The data were processed using the XCMS package in R software v. 3.1.0 for peak detection, alignment, and integration. The optimized XCMS parameters were as follows: method $=$ cent wave, signal-to-noise threshold $($ sn thresh $)=10$, maximum $m / z$ deviation tolerance $(\mathrm{ppm})=30$, peak width $=(10,30)$, bandwidth $(\mathrm{bw})=10$, and integration method $=1$ (default). Other parameters were set to the default values. The peaks of isotopes and adducts were annotated using the CAMERA package. The matrix $(\mathrm{m} /$ $z$-retention time-intensity pairs) was generated and exported to a CSV 
file. Microsoft Excel ${ }^{\mathrm{TM}} 2010$ was used to remove isotopes and filter the data according to the $80 \%$ rule and a noise cut-off value of 10,000 . The data were subjected to multivariate statistical analysis using SIMCA-P11.5 software (Umetris AB, Umea, Sweden); all variables were Pareto-Scaled $[(\mathrm{X}-\bar{X}) / \sqrt{ } \overline{S D}, \bar{X}$ : Mean, SD: standard deviation]. For data normalization, the LOESS fitting method was used for plasma samples and the MSTUS data normalization for urine samples using $\mathrm{R}$ and NOREVA (http://server.idrb.cqu.edu.cn/noreva/). A principle component analysis (PCA) was performed to visualize patterns and groupings, and an orthogonal partial least squares-discriminant analysis (OPLS DA) was performed to identify variables that could be used to distinguish the patients with breast cancer from the healthy controls based on their variable importance in projection values.

\section{Statistical analysis}

Statistical analysis was performed using Statistical Package for the Social Sciences (SPSS) v. 23.0 software (IBM Corp., Armonk, USA). The nonparametric Kruskal-Wallis and Mann-Whitney U-tests were used to evaluate the significance of differences between the patients with breast cancer and the healthy controls. A $p$-value of $<0.05$ was considered indicative of statistical significance. To evaluate the sensitivity and specificity of biomarkers, receiver operating characteristic (ROC) curves were plotted using MedCalc software v. 16.4.3 (MedCalc Software BVBA, Ostend, Belgium).

\section{RESULTS}

\section{Clinicopathological characteristics of the study}

\section{population}

The study population comprised 14 patients with breast cancer and 16 healthy controls. The mean age of the patients with breast cancer was $58.6 \pm 10.7$ years (range, $42-77$ years) and that of the healthy controls was $56.1 \pm 2.0$ years (range, $52-59$ years). Among the 14 patients with breast cancer, two (14.3\%), four (28.6\%), and eight (57.2\%) had a low, intermediate, and high histologic grade, respectively. In terms of tumor size and lymph node (LN) status, eight patients (57.1\%) had tumors larger than $2 \mathrm{~cm}$ and six patients (42.9\%) had LN metastasis. Regarding hormone receptor expression, eight tumors (57.1\%) were positive for estrogen receptor (ER) and seven tumors (50.0\%) were positive for progesterone receptor (PR). Table 1 shows the clinicopathologic
Table 1. The clinicopathological data of breast cancer patients in this study

\begin{tabular}{|c|c|}
\hline Characteristic & №. (\%) \\
\hline \multicolumn{2}{|l|}{ Age $(y r)^{*}$} \\
\hline Healthy $(n=16)$ & $56.1 \pm 2.0(52-59)$ \\
\hline Cancer $(n=14)$ & $58.6 \pm 10.7(42-77)$ \\
\hline \multicolumn{2}{|l|}{ Histologic grade } \\
\hline Low & $2(14.3)$ \\
\hline Intermediate & $4(28.6)$ \\
\hline High & $8(57.1)$ \\
\hline \multicolumn{2}{|l|}{ Tumor size $(\mathrm{cm})$} \\
\hline$\leq 2$ & $8(57.1)$ \\
\hline$>2$ & $6(42.9)$ \\
\hline \multicolumn{2}{|l|}{ LN metastasis } \\
\hline Negative & $8(57.1)$ \\
\hline Positive & $6(42.9)$ \\
\hline \multicolumn{2}{|l|}{ ER } \\
\hline Negative & $6(42.9)$ \\
\hline Positive & $8(57.1)$ \\
\hline \multicolumn{2}{|l|}{ PR } \\
\hline Negative & $7(50.0)$ \\
\hline Positive & $7(50.0)$ \\
\hline \multicolumn{2}{|l|}{ HER2 } \\
\hline Negative & $11(78.6)$ \\
\hline Positive & $3(21.4)$ \\
\hline \multicolumn{2}{|l|}{ Triple negativity } \\
\hline Yes & $3(21.4)$ \\
\hline No & $11(78.6)$ \\
\hline \multicolumn{2}{|l|}{ Ki-67 } \\
\hline Low & $2(14.3)$ \\
\hline Intermediate & $5(35.7)$ \\
\hline High & $7(50.0)$ \\
\hline \multicolumn{2}{|l|}{ T stage } \\
\hline $\mathrm{T} 1$ & $6(42.9)$ \\
\hline $\mathrm{T} 2$ & $7(50.0)$ \\
\hline T3 & $1(7.1)$ \\
\hline $\mathrm{T} 4$ & 0 \\
\hline \multicolumn{2}{|l|}{ N stage } \\
\hline No & $8(57.1)$ \\
\hline N1 & $2(14.3)$ \\
\hline N2 & $2(14.3)$ \\
\hline N3 & $2(14.3)$ \\
\hline \multicolumn{2}{|l|}{ TNM stage } \\
\hline I & $5(35.7)$ \\
\hline$\|$ & $5(35.7)$ \\
\hline III & $4(28.6)$ \\
\hline
\end{tabular}

$\mathrm{LN}=$ lymph node; $\mathrm{ER}=$ estrogen receptor; $\mathrm{PR}=$ progesterone receptor; Ki67: low for $<10 \%$, intermediate for $10-20$, high for $>20 \%$ tumor cells. *Mean \pm SD (range).

characteristics of the patients with breast cancer.

Urinary metabolite profiles of the patients with breast cancer

A comparison of the metabolite concentrations in the urine of the 


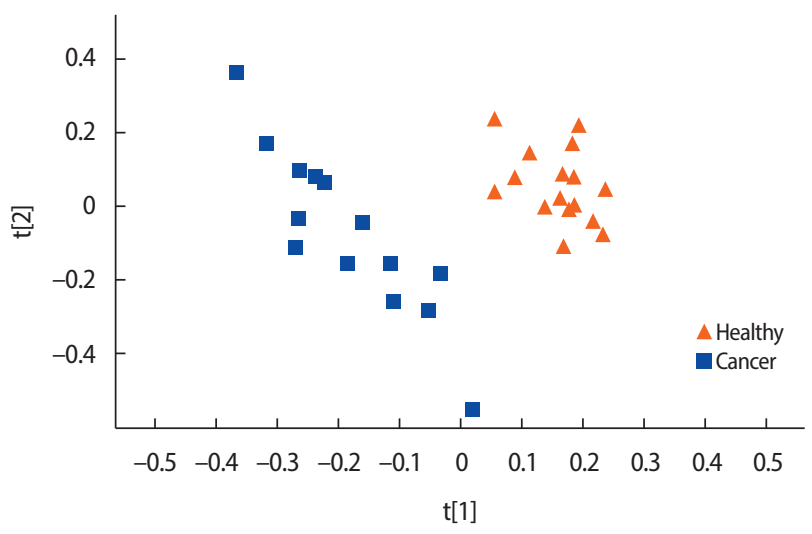

Figure 1. OPLS-DA score plots of the patients with breast cancer and the healthy controls, based on metabolite profiles. Blue squares and black triangles represent breast cancer patients and healthy controls, respectively.

healthy controls $(n=16)$ and the patients with breast cancer $(n=14)$ revealed substantial differences. An OPLS-DA with one predictive component and two orthogonal components showed clear separation between the groups. All the healthy controls were scattered on the right side and most of the patients with breast cancer on the left side (Figure 1).

\section{Differences in the concentrations of urinary metabolites}

In total, 95 metabolites with concentration differentials were identified. Among these, 24 candidate biomarkers, comprising 10 amino acids, 10 organic compounds, two carbohydrates, a xenobiotic, an energy-related metabolite, and a nucleotide, were subjected to foldchange analysis (Table 2, Figure 2). Student's t-test revealed that the concentrations of 7 and 17 of these 24 metabolites were significantly higher and lower, respectively, in the patients with breast cancer than in the healthy controls $(p<0.05)$ (Tables 3, 4). For example, the concentration of $\mathrm{N}$-glycine was 13.83 fold higher in the patients with breast cancer than in the healthy controls.

\section{ROC analysis of urinary metabolites}

ROC analysis was performed on the three metabolites that exhibited the greatest differences in concentration between the patients with breast cancer and the healthy controls. Among the metabolites with relatively high levels in the patients with breast cancer, $\mathrm{N}$-(2-furoyl) glycine had the highest AUC value (0.902) for discriminating between patients with breast cancer and healthy controls. Figures 3 and 4 show the ROC curves of the three metabolites with the greatest concentra-
Table 2. Fold changes of biomarker candidates in the patients with breast cancer compared to the healthy controls

\begin{tabular}{|c|c|}
\hline Metabolite & Fold change \\
\hline \multicolumn{2}{|l|}{ Amino acid } \\
\hline 5-oxoproline (pyroglutamic acid) & 0.76 \\
\hline 7-Methylguanine & 0.60 \\
\hline Creatinine & 0.42 \\
\hline Histidine & 2.61 \\
\hline Kynurenic acid & 0.77 \\
\hline L-Tryptophan & 0.67 \\
\hline $\mathrm{N}$-(2-Furoyl)glycine & 13.83 \\
\hline N-Acetylneuraminic acid & 0.47 \\
\hline Spermidine & 0.50 \\
\hline \multicolumn{2}{|l|}{ Organic compounds } \\
\hline$( \pm)$-Sulfobutanedioic acid & 1.58 \\
\hline D-Tagatose & 2.47 \\
\hline D-Xylulose & 0.68 \\
\hline Glutaminyl-Gamma-Glutamate & 0.75 \\
\hline L-Galacto-2-heptulose & 0.39 \\
\hline N-Acetylgalactosamine 6-sulfate & 0.47 \\
\hline Phenol sulphate & 1.58 \\
\hline Trigonellinamide & 0.29 \\
\hline Tyrosine & 0.74 \\
\hline Salicyluric acid & 0.44 \\
\hline \multicolumn{2}{|l|}{ Carbohydrates } \\
\hline Gluconic acid & 1.88 \\
\hline Sorbitol & 1.60 \\
\hline \multicolumn{2}{|l|}{ Xenobiotics } \\
\hline 1-Methyluric acid & 0.60 \\
\hline \multicolumn{2}{|l|}{ Energy } \\
\hline Malic acid & 0.59 \\
\hline \multicolumn{2}{|l|}{ Nucleotides } \\
\hline Xanthine & 0.53 \\
\hline
\end{tabular}

24 candidate biomarkers with significant $p$-value $(<0.05)$ were shown among 95 identified metabolites.

tion differentials (AUCs of $\geq 0.7$ and $\geq 0.8$, respectively), and their AUCs are listed in Tables 3 and 4.

\section{DISCUSSION}

Several metabolomics studies of urine have been reported, typically based on both nuclear magnetic resonance and MS; however, most metabolomics studies of breast cancer have relied on MS [9]. The following urine metabolomics studies have identified biomarkers for breast cancer. Chen et al. [8] were the first to examine the metabolite profiles of the urine of healthy volunteers and patients with breast cancer, and they identified 12 metabolites — including amino acids, organic acids, and nucleosides — as potential biomarkers. Henneges et 


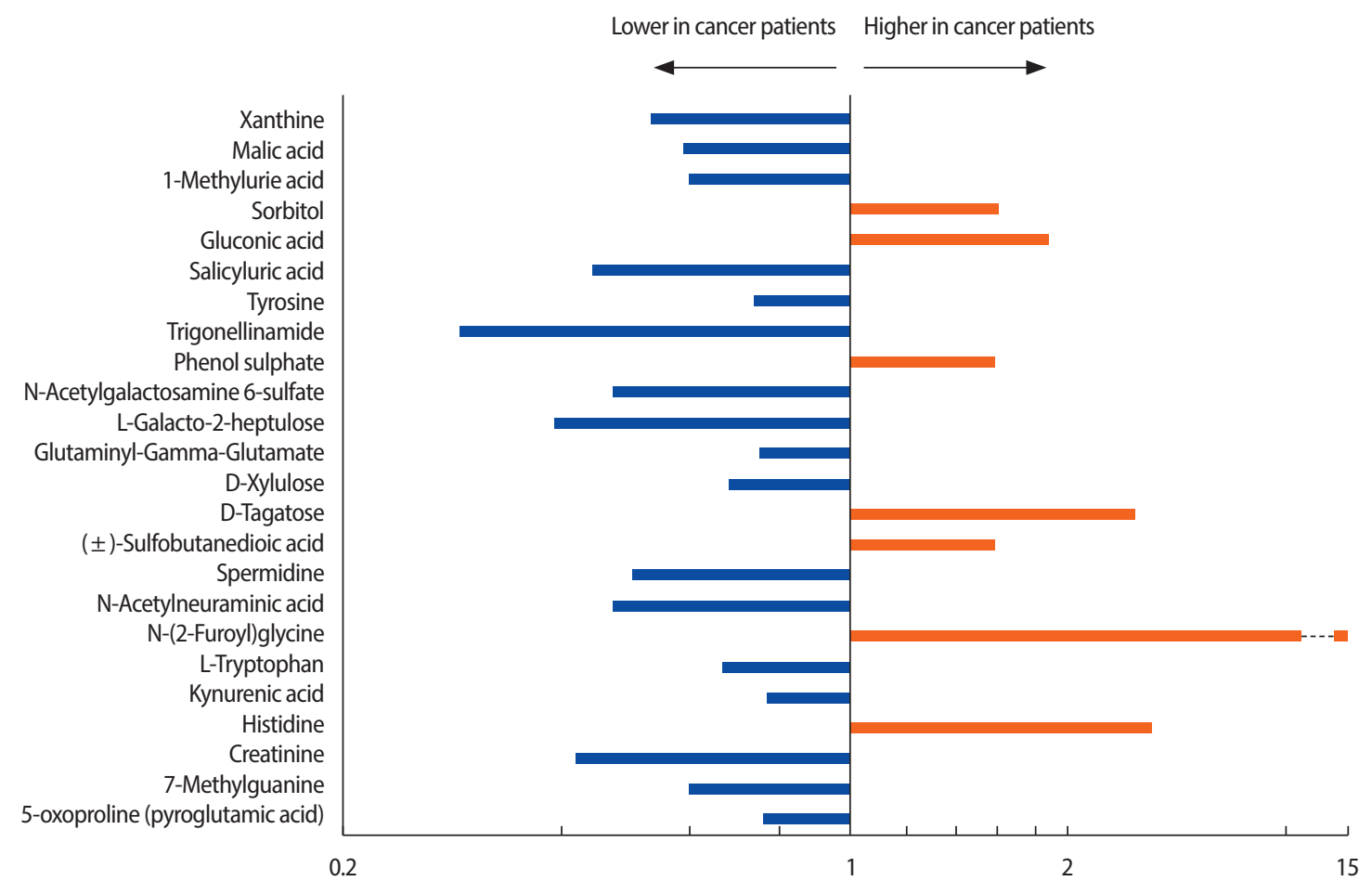

Figure 2. Fold changes of 24 metabolites whose concentrations were significantly higher and lower in the patients with breast cancer compared to the healthy controls.

Table 3. Fold changes and AUC of metabolites whose concentrations were significantly increased in the patients with breast cancer compared to the healthy controls

\begin{tabular}{lcccc}
\hline Metabolite & Fold change & $p$-value & AUC & $p$-value \\
\hline N-(2-Furoyl)glycine & 13.83 & 0.001 & 0.902 & 0.001 \\
Histidine & 2.61 & 0.005 & 0.799 & 0.005 \\
D-Tagatose & 2.47 & 0.031 & 0.732 & 0.031 \\
Gluconic acid & 1.88 & 0.014 & 0.656 & 0.146 \\
Sorbitol & 1.60 & 0.038 & 0.763 & 0.014 \\
( \pm )-Sulfobutanedioic acid & 1.58 & 0.031 & 0.732 & 0.031 \\
Phenol sulphate & 1.58 & 0.042 & 0.719 & 0.042 \\
\hline
\end{tabular}

al. [11] analyzed urine samples from 85 patients with breast cancer and healthy controls by LC-MS. A computational analysis yielded 44 pairwise ratios of metabolite features with a sensitivity of $83.5 \%$ and a specificity of $90.6 \%$ for best prediction of breast cancer. Nam et al. [12] identified homovanillate, 4 hydroxyphenylacetate, 5 hydroxyindoleacetate, and urea in urine as biomarkers of breast cancer by GCMS in 50 patients with breast cancer and 50 normal controls. Although these previous studies were similar to our study, we focused on detecting differences in the concentrations of metabolites in urine by LC-MS.

The concentrations of most metabolites in urine are reported to be
Table 4. Fold changes and AUC of metabolites whose concentrations were significantly decreased in the patients with breast cancer compared to the healthy controls

\begin{tabular}{lcccc}
\hline Metabolite & Fold change & $p$-value & AUC & $p$-value \\
\hline Trigonellinamide & 0.29 & 0.001 & 0.897 & 0.001 \\
L-Galacto-2-heptulose & 0.39 & 0.003 & 0.817 & 0.003 \\
Creatinine & 0.42 & 0.003 & 0.821 & 0.003 \\
Salicyluric acid & 0.44 & 0.001 & 0.857 & 0.001 \\
N-Acetylgalactosamine & 0.47 & 0.002 & 0.839 & 0.002 \\
$\quad$ 6-sulfate & & & & \\
Spermidine & 0.50 & 0.001 & 0.844 & 0.001 \\
Xanthine & 0.53 & 0.001 & 0.938 & 0.001 \\
Malic acid & 0.59 & 0.038 & 0.732 & 0.038 \\
1-Methyluric acid & 0.60 & 0.022 & 0.746 & 0.022 \\
L-Tryptophan & 0.67 & 0.014 & 0.763 & 0.014 \\
D-Xylulose & 0.68 & 0.001 & 0.844 & 0.001 \\
7-Methylguanine & 0.70 & 0.009 & 0.781 & 0.009 \\
Tyrosine & 0.74 & 0.046 & 0.714 & 0.046 \\
Glutaminyl-Gamma- & 0.75 & 0.042 & 0.719 & 0.042 \\
$\quad$ Glutamate & & & & \\
5-oxoproline (pyroglutamic & 0.76 & 0.001 & 0.844 & 0.001 \\
$\quad$ acid) & & & & \\
Kynurenic acid & 0.77 & 0.042 & 0.719 & 0.042 \\
N-Acetylneuraminic acid & 0.77 & 0.010 & 0.777 & 0.010 \\
\hline$\quad$
\end{tabular}

lower in patients with breast cancer than in normal controls [13], with seven exceptions. Remarkably, the concentration of N-(2-furoyl) gly- 

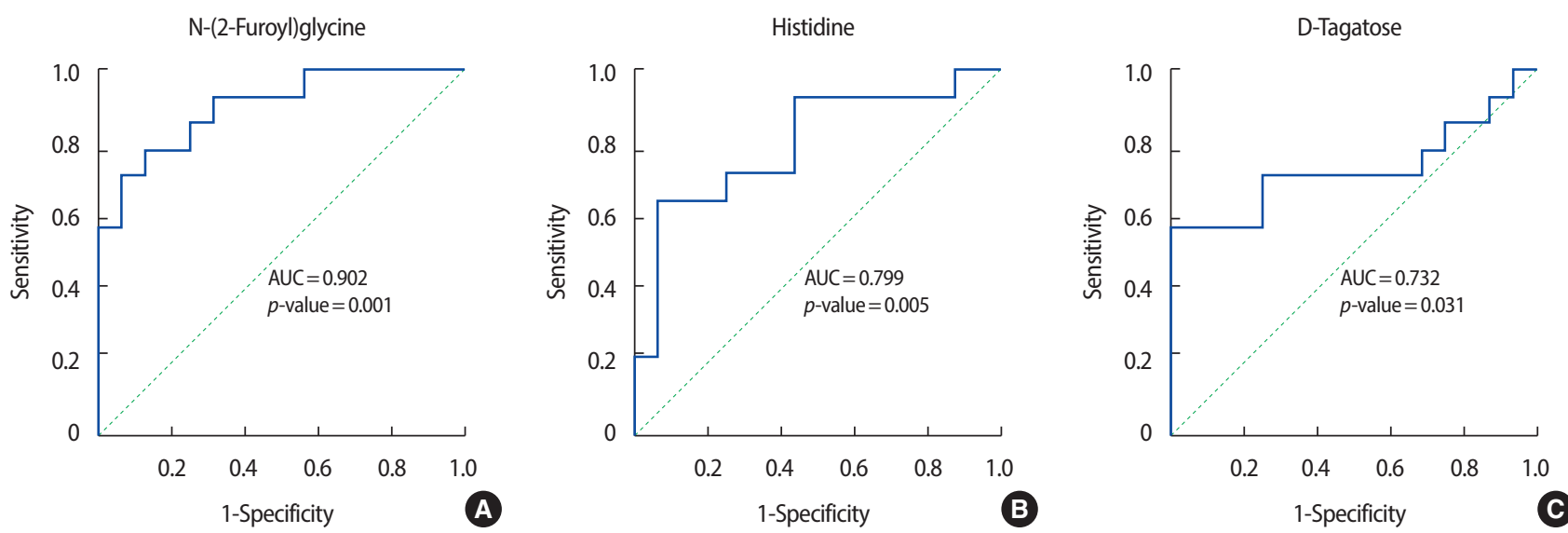

Figure 3. ROC curves of three metabolites whose concentrations were higher in the patients with breast cancer compared to the healthy controls.
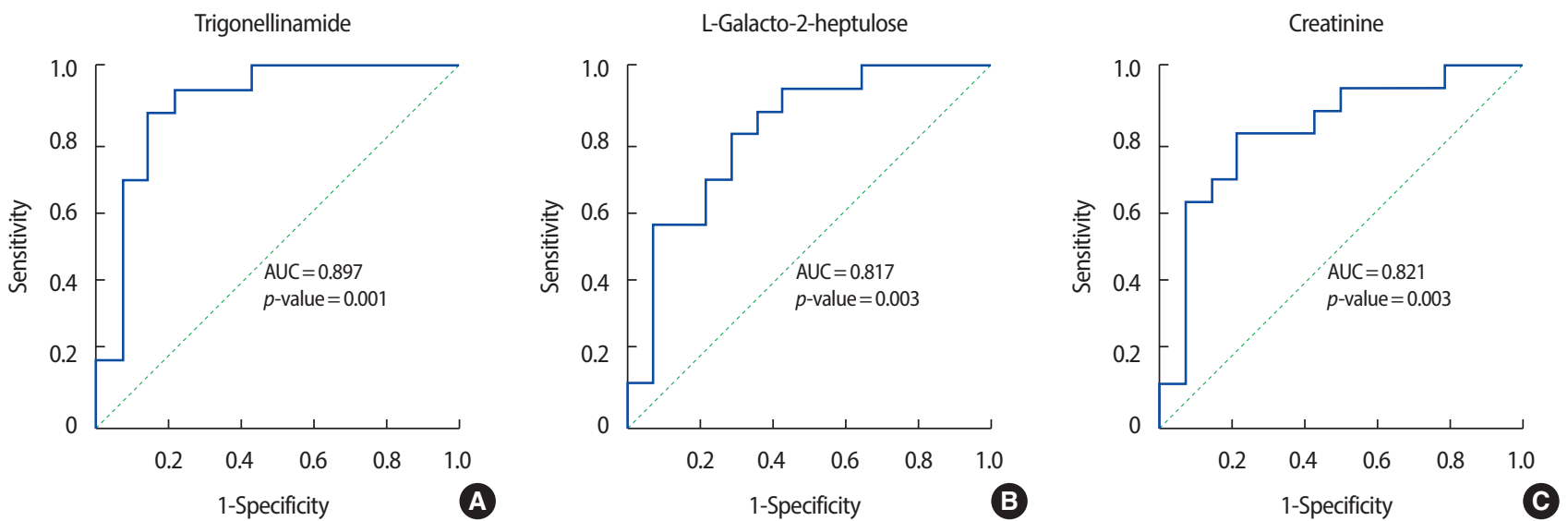

Figure 4. ROC curves of three metabolites whose concentrations were lower in the patients with breast cancer compared to the healthy controls.

cine, a minor metabolite of fatty acids, was 13 -fold higher in the patients with breast cancer than in the healthy controls (AUC, 0.902). The concentration of this metabolite can be affected by food intake and is involved in mitochondrial fatty-acid $\beta$-oxidation. Therefore, the increased concentration of $\mathrm{N}$-(2-furoyl) glycine suggests that $\beta$-oxidation of fatty acids is enhanced in patients with breast cancer, consistent with reports that lipolysis and lipid oxidation are upregulated in cancer cells $[14,15]$. Fatty acids are a good source of fuel for cancer cells because mitochondrial fatty-acid oxidation produces more adenosine triphosphate (ATP) than does oxidation of, for instance, glucose or amino acids [16].

In addition to N-(2-furoyl) glycine, the differential concentrations of histidine, xanthine, and pyroglutamic acid in the patients with breast cancer and in the healthy controls were consistent with previous reports. Concentrations of histidine, an essential amino acid in humans and other mammals, were higher in the patients with breast cancer than in the healthy controls, in agreement with a previous report on metabolites in blood [17]. The decreased concentrations of tricarboxylic acid (TCA)-cycle intermediates, such as malic acid, is suggestive of a suppressed TCA cycle in the patients with breast cancer. Similarly, the concentrations of several TCA-cycle intermediates in urine have been reported to be lower in patients with colorectal cancer compared to controls [18].

The decreased xanthine concentration (AUC, 0.938) in the patients with breast cancer is noteworthy. Xanthine is a purine base synthesized from hypoxanthine, which is oxidized to xanthine and subsequently to uric acid by xanthine oxidase with production of reactive oxygen species [19]. Purine nucleotides are synthesized de novo or by salvaging of catabolized purine bases, principally xanthine. The salvage pathway is more efficient than de novo synthesis in terms of ATP 
consumption [20]. This metabolic change is an important driver of the proliferation of cancer cells because it promotes efficient production of ATP. In this study, the low levels of xanthine in the urine of the patients with breast cancer indicate enhanced consumption of that compound, implying that in breast cancer cells the purine biosynthesis pathway is substituted for the salvage pathway.

Levels of pyroglutamic acid, also known as 5-oxoproline (the cyclic lactam of glutamic acid), were lower in the patients with breast cancer than in the healthy controls. Glutamic acid and glutamine are inter-convertible. Glutamine plays important roles in cancer cells by acting as a nitrogen donor in the synthesis of nucleotides and amino acids. Glutamine also assists in the uptake of essential amino acids and is involved in the activation of TOR kinases [6]. Glutamine is reportedly depleted in the skeletal muscle of cancer patients due to its consumption by cancer cells [21]. Exogenous glutamine is an important source of energy and molecular building blocks for many tumors [22]. Therefore, our findings imply that, in the patients with breast cancer, pyroglutamic acid was consumed to supply the energy required for proliferation of tumor cells. Although further studies of its metabolism are warranted, pyroglutamic acid may be a promising diagnostic biomarker and therapeutic target for breast cancer.

To investigate their performance as biomarkers, six metabolites were subjected to ROC analysis; all showed an AUC of $\geq 0$.7. However, this finding must be validated using independent datasets, which may have distinct characteristics. This study was limited by the small sample size. We plan to conduct a comparative analysis of blood and urine specimens with a larger number of subjects.

Even though the advent of metabolomics has been facilitated by improvements in instrument technology, it has some significant limitations. Metabolites are sensitive to environmental impacts. They are affected by environmental factors of the subjects including diet, alcohol, medication and physical activity [23]. In addition, chronic diseases or conditions such as obesity, diabetes and cardiovascular disease, in which metabolic dysregulation plays a key role in pathophysiology and disease progression, are variables in metabolism [24]. Furthermore, some metabolites are labile and are of extremely low abundance in the human body. Protocols for extracting and obtaining the metabolome have been well developed for various biological samples, including tissue, plasma and urine. Although anyone can use these protocols easily, even minor changes in procedure can result in a major impact on the observed metabolome. For example, subtle change of temperature can affect activity of enzyme, distorting concentration of metabolites. Because of this chemical instability, improper handling of the samples may act as biases without producing consistent results [25].

In this study, the patients with breast cancer had a urinary metabolite profile that was distinct from that of the healthy controls. Diagnostic screening based on urine samples is non-invasive, inexpensive, and easy to perform. Measurement of the concentrations of metabolites in urine may enable early detection of breast cancer and so increase the survival rate by facilitating timely application of treatment. We plan to explore biomarkers of breast cancer further by combining the findings from this study with biomarkers in blood identified by LC-MS.

\section{ACKNOWLEDGMENTS}

The breast cancer patients' plasma samples and data were obtained from the Inje Biobank at the Inje University Busan Paik Hospital, Republic of Korea.

The biospecimens and data used for this study were provide by the Biobank of Chungnam National University Hospital, a member of the Korea Biobank Network.

\section{REFERENCES}

1. Skaane P. Studies comparing screen-film mammography and fullfield digital mammography in breast cancer screening: updated review. Acta radiol 2009;50:3-14.

2. Vander Heiden MG. Targeting cancer metabolism: a therapeutic window opens. Nat Rev Drug Discov 2011;10:671.

3. Warburg O. On the origin of cancer cells. Science 1956;123:309-14.

4. Koppenol WH, Bounds PL, Dang CV. Otto Warburg's contributions to current concepts of cancer metabolism. Nat Rev Cancer 2011;11:325.

5. Hsu PP, Sabatini DM. Cancer cell metabolism: warburg and beyond. Cell 2008;134:703-7.

6. Wise DR, Thompson CB. Glutamine addiction: a new therapeutic target in cancer. Trends Biochem Sci 2010;35:427-33.

7. Altman BJ, Stine ZE, Dang CV. From Krebs to clinic: glutamine me- 
tabolism to cancer therapy. Nat Rev Cancer 2016;16:619.

8. Chen Y, Zhang R, Song Y, He J, Sun J, Bai J, et al. RRLC-MS/MSbased metabonomics combined with in-depth analysis of metabolic correlation network: finding potential biomarkers for breast cancer. Analyst 2009;134:2003-11.

9. Günther UL. Metabolomics biomarkers for breast cancer. Pathobiology 2015;82:153-65.

10. Johnson C, Manna S, Krausz K, Bonzo J, Divelbiss R, Hollingshead $\mathrm{M}$, et al. Global metabolomics reveals urinary biomarkers of breast cancer in a MCF-7 xenograft mouse model. Metabolites 2013;3: 658-72.

11. Henneges C, Bullinger D, Fux R, Friese N, Seeger H, Neubauer H, et al. Prediction of breast cancer by profiling of urinary RNA metabolites using support vector machine-based feature selection. BMC Cancer 2009;9:104.

12. Nam H, Chung BC, Kim Y, Lee K, Lee D. Combining tissue transcriptomics and urine metabolomics for breast cancer biomarker identification. Bioinformatics 2009;25:3151-7.

13. Slupsky CM, Steed H, Wells TH, Dabbs K, Schepansky A, Capstick $\mathrm{V}$, et al. Urine metabolite analysis offers potential early diagnosis of ovarian and breast cancers. Clin Cancer Res 2010;16:5835-41.

14. Min HK, Kong G, Moon MH. Quantitative analysis of urinary phospholipids found in patients with breast cancer by nanoflow liquid chromatography-tandem mass spectrometry: II. Negative ion mode analysis of four phospholipid classes. Anal Bioanal Chem 2010;396:1273-80

15. Melone MAB, Valentino A, Margarucci S, Galderisi U, Giordano A, Peluso G. The carnitine system and cancer metabolic plasticity. Cell Death Dis 2018;9:228.
16. Liu Y. Fatty acid oxidation is a dominant bioenergetic pathway in prostate cancer. Prostate Cancer Prostatic Dis 2006;9:230.

17. Jobard E, Pontoizeau C, Blaise BJ, Bachelot T, Elena-Herrmann B, Trédan O. A serum nuclear magnetic resonance-based metabolomic signature of advanced metastatic human breast cancer. Cancer Lett 2014;343:33-41.

18. Qiu Y, Cai G, Su M, Chen T, Liu Y, Xu Y, et al. Urinary metabonomic study on colorectal cancer. J Proteome Res 2010;9:1627-34.

19. Thamer NA. Detection of xanthine oxidase in breast cancer. Iraqi J Cancer Med Genet 2018;6.

20. Kondo M, Yamaoka T, Honda S, Miwa Y, Katashima R, Moritani M, et al. The rate of cell growth is regulated by purine biosynthesis via ATP production and G1 to S phase transition. J Biochem 2000;128: $57-64$.

21. Kulkarni C, Kulkarni K, Hamsa BJ. L-Glutamic acid and glutamine: exciting molecules of clinical interest. Indian J Pharmacol 2005;37: $148-54$.

22. Budczies J, Pfitzner BM, Györffy B, Winzer KJ, Radke C, Dietel M, et al. Glutamate enrichment as new diagnostic opportunity in breast cancer. Int J Cancer 2015;136:1619-28.

23. Scalbert A, Brennan L, Fiehn O, Hankemeier T, Kristal BS, van Ommen B, et al. Mass-spectrometry-based metabolomics: limitations and recommendations for future progress with particular focus on nutrition research. Metabolomics 2009;5:435.

24. Newgard CB. Metabolomics and metabolic diseases: where do we stand? Cell Metab 2017;25:43-56

25. Riekeberg E, Powers R. New frontiers in metabolomics: from measurement to insight. F1000Research 2017;6:1148. 